\title{
Human Face and Facial Parts Detection using Template Matching Technique
}

\author{
Payal Bose, Samir Kumar Bandyopadhyay
}

\begin{abstract}
Face recognition has become relevant in recent years because of its potential applications. The aim of this paper is to find out the relevant techniques which give not only better accuracy also the efficient speed. There are several techniques available for face detection which give much better accuracy but the execution speed is not efficient. In this paper, a normalized cross-correlation template matching technique is used to solve this problem. According to the proposed algorithm, first different facial parts are detected likes mouth, eyes, and nose. If any of the two facial parts are found successfully then the face can be detected. For matching the templates with the target image, the template rotates at a certain angle interval.

Keywords: Face detection, Face Recognition, Template Matching, Normalized Cross-Correlation
\end{abstract}

\section{INTRODUCTION:}

Face Detection and its recognition one of the widely used technology with today's growing information technology world. It is one of the hottest topics in the artificial intelligence and pattern recognition field because of its application in the field of public security, system authentication, medical fields and so on. But today due to different kinds of face poses and different illumination factors it is very difficult to detect and identify the face and its facial features accurately. Template matching is a very common task of pattern recognition and a very high-level machine vision technique. It allows identifying a part of single or multiple images that matches a specific image pattern. In template matching two primary components are required, (I) Template Image: It is a piece of an image that we need to find in the source image. Here the goal is to find the best matching area in the test image. (II) Source Image: It is the image we need to find the best-matched location of the template image. Face detection is the first state of face recognition and further detection. In pattern recognition and computer vision technology, face detection has the most important value. Template matching is the best solution in pattern recognition technique, to find the best matching area for face detection. Based on the various approach face detection process classified into four categories i) Knowledge-Based, ii) Feature-Based, iii) Template-Based and iv) Appearance-Based. In this paper, a template-based face detection technique used to identify the human face along with its different facial parts like eyes, nose, and mouth.

Revised Manuscript Received on April 27, 2020.

* Correspondence Author

Payal Bose*, Ph.D. Research Scholar of Lincoln University College, Malaysia

Prof. Samir Kumar Bandyopadhyay, Distinguish Professor, Lincoln University College, Malaysia

(C) The Authors. Published by Blue Eyes Intelligence Engineering and Sciences Publication (BEIESP). This is an open access article under the CC BY-NC-ND license (http://creativecommons.org/licenses/by-nc-nd/4.0/)

\section{REVIEW ON EXISTING WORK:}

Face detection and recognition is one of the challenging tasks. There are several methods are available for face detection and recognition. But still, it is one of the most important tasks. Because face detection is very much important for security sections in different places, crowd analysis and many more. There are several authors describe several techniques for face detection. In 2017, the authors, Shrinath Oza, Abdullah Khan, Nilabh Swapnil, Aayush Sharma, Jayashree Chaudhari, implemented an algorithm ${ }^{[1]}$, the Restricted Boltzmann machine with the rules of deep learning and deep belief network for face recognition. According to this algorithm, it stores the face images as input in a database and a neural network used to ensure the efficiency and improvement of the recognition system trained the neural network greedy layer-wise. In 2018, the authors, Sang-II Choi, Sung-Sin Lee, Sang Tae Choi, WonYong Shin ${ }^{[2]}$ construct a composite feature vector for face recognition based on discriminant analysis. According to the method, they first extract the holistic features and the local features from the whole face image and use various types of local images for discriminant feature extraction. Then measure the amount of discriminative information in the individual holistic features and local features and construct a composite features for face recognition. In 2019, author Ru Wang and Xinshi Hi proposed a face recognition method by using template matching and neural networks ${ }^{[3]}$. In the year 2017 the authors Namrata Singh, A. K. Daniel and Pooja Chaturvedi proposed a method to detect different facial parts using $\mathrm{YCbCr}$ Skin color segmentation and template matching techniques to detect the faces ${ }^{[4]}$. In the year 2018, authors Chen Yan1, Zhengqun Wang1, Yang Yang1, Chunlin Xu2 describe a method that detects the multi-angle face based on Gaussian Mixture Model and Improved Template Matching ${ }^{[5]}$.

\section{Different Face Detection Techniques:}

Based on the various face detection approach, this process classified into four categories.

\section{1) Knowledge-Based:}

Knowledge-based method is a form of artificial intelligence and its aim to capture human knowledge and make an ultimate decision. To make a decision it depends on a set of rules. This approach is actually a rule-based system and the rules may vary with respect to the problem-solving approach. Example: A face contains eyes, nose, mouth and they all have a certain distance with each other. The big problem with these kinds of approaches is to create appropriate rules. If the rules are not appropriate then there could be many false-positive detections create.

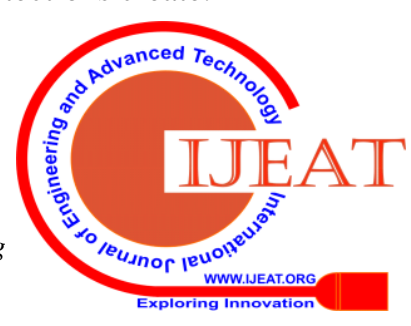


This approach is also not properly applicable to detect more than one face in multiple images.

\section{2) Feature-Based:}

The feature-based approach extracts various structural features from the image. Then a classifier used to classify the known image features and stored them in a database. Now based on these known structural features the same classifier classifies the unknown images. This idea overcomes the instinctive knowledge of classifying an image. In this approach, the successive rate is sufficiently high.

\section{3) Appearance-Based:}

The appearance-based approach based on recording the statistical value of pixels in an image. Basically in this approach, a set of training datasets used to record the statistical value and then these values are used to detect the target goal. Various appearance-based techniques are available to detect faces in an image. The appearance-based method based on the techniques of statistical analysis and try to find out the relevant data from the sets of image. This technique also used feature extraction for face detection.

\section{4) Template-Based:}

Template matching[6] is a high-level machine vision technique and a very common and important task in pattern recognition. This technique allows identifying single or multiple parts of an image that matches with the predeclared image pattern. This matching algorithm depends on the nature of the image and the problem that needs to solve. Based on this template matching techniques divided into two categories.

\subsection{1) Feature-Based Matching:}

When both the test image[7] and template image contain more than one correspondence with respect to its feature and control points then this kind of matching technique is used. In this approach, the aim is to find the pair-wise connection between target and source image. Here the spatial relation is used to perform the matching. For extracting the matching features spatial relation, invariant descriptor, wavelet different kinds of techniques used.

\subsection{2) Area-Based Matching:}

This type of matching technique suitable when the template has no strong features with the target image, it provides directly matching over the image pixels. Some times direct matching template and the target image are impossible then the eigenvalue and eigen-spaced are used for matching. These values give detailed information about the image under various conditions, like illuminated image, color contrast image, etc for matching. Some times this kind of technique divided the target image into several sub-images for better matching performance. To perform the template matching there are two types of method available,

\section{i) Naive Template Matching:}

This is a basic template matching method. In this method, a template is created which is identical with the target image the with or without scaling the target image scanned by the template image. Finally, it returns the strongest similar pattern which is matched with the template image. Fig-1 shows the example of this matching where the Naive template matching technique used to extract the template from the target image.

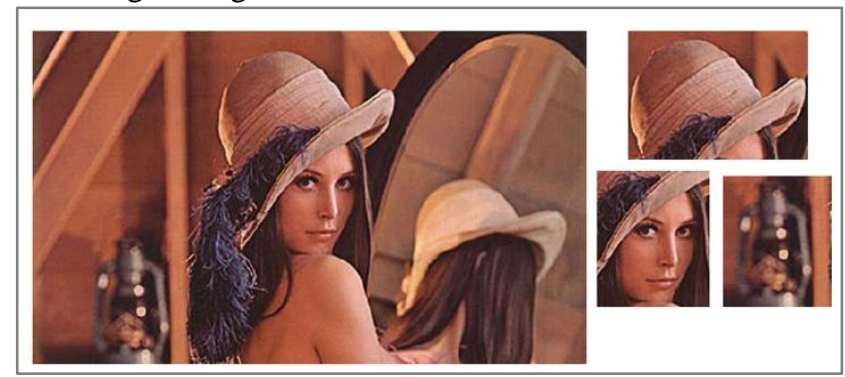

Fig:1) (Picture of Lena) Left side: target image, right side: template images.

The relation metric between the target (f) and the template (t) image is given by,

Sum of Squared Diffrences (SSD)

$$
=\sum_{x, y}[f(x, y)-t(x-u, y-v)]^{2}
$$

Where, $\mathrm{x}, \mathrm{y}, \mathrm{u}, \mathrm{v}$ are the pixel values of target and template images respectively.

\section{ii) Image Correlation Matching:}

In this method, the similarity between target and template image needs to calculate. For fiding the similarity, the pattern must be aligned properly. The similarity metric measure here by finding the correlation between target and template images.

\section{a) Cross-Correlation:}

When the two image size is different the finding the similarity metric between them cross-correlation matching is used. This method is similar to the convolution between two functions. The cross-correlation is defined as:

CrossCorrelation(Image1, Image 2)

$$
\begin{aligned}
& =\sum_{x, y}[\operatorname{Image1}(x, y) \\
& \times \operatorname{Image} 2(x, y)]
\end{aligned}
$$

\section{b) Normalized Cross-Correlation:}

To improve the cross-correlation matching the normalized cross-correlation[8] matching is used. It is the updated version of cross-correlation matching. The main reasons behind using normalized cross-correlation are,

i) The normalized cross-correlation remains unchanged when the global brightness change that means the image intensity variations have much less effect on the correlation matric.

The correlation value of two images varies from $[-1,+1]$, whereas for normalized correlation in only tends to +1 , which is easy to understand.

The equation of normalized cross-correlation is defined as,

Normalized Cross Correlation(NCC)

$$
=\frac{\sum_{x, y}\left[f(x, y)-\bar{f}_{u, v}\right][t(x-u, y-v)-\bar{t}]}{\left[\sum_{x, y}\left[f(x, y)-\bar{f}_{u, v}\right]^{2} \sum_{x, y}[t(x-u, y-v)-\bar{t}]^{2}\right]^{0.5}}
$$

Here $(x, y)$ is the original image; $\bar{f}$ is the mean of image intensity in the region under the template, $t$ is the template and $\bar{t}$ represents the mean of image intensity in the template. Here $(\mathrm{x}, \mathrm{y})$ and $(u, \mathrm{v})$ represent pixel coordinates.

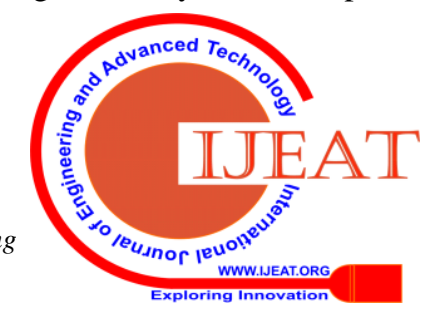




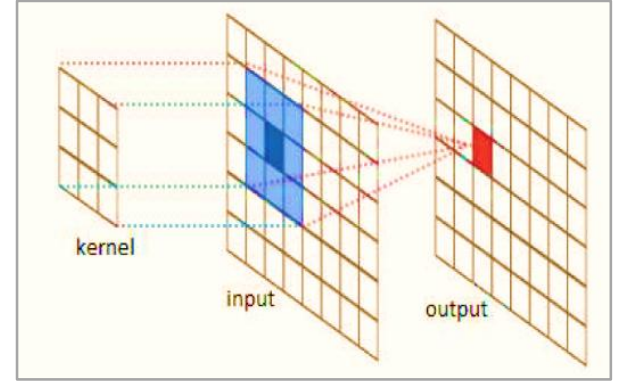

Fig: 2) Object Recognition using Template Matching Technique

2. Proposed Work for Face and Facial Parts Detection: In this paper, the main goal is to find out the human face along with its different facial parts like eyes, nose, and mouth from a group of people images.

Therefore, to find out the face and facial parts of a human normalized cross-correlation template matching algorithm used in a different manner. Here to solve the above problem, that means to found out the human face and its facial parts', it is needed to create templates for eyes, nose, mouth, and as well as for the faces to match. For eyes nose, mouth and face detection first we need to create a template database for each one with a fixed-sized. No template has a different size here. The sample of eyes, nose and mouth template are shown below.

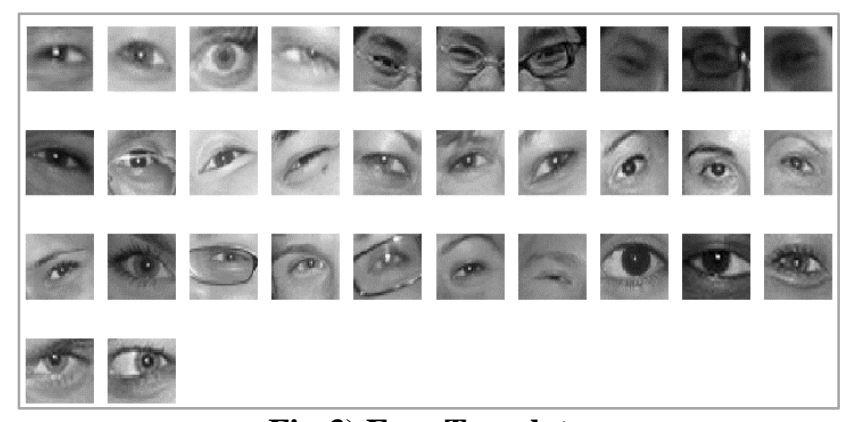

Fig:3) Eyes Template

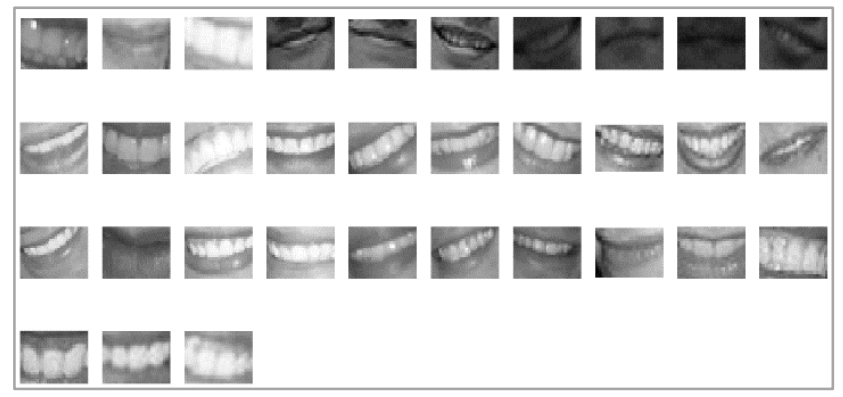

Fig:4) MouthTemplate

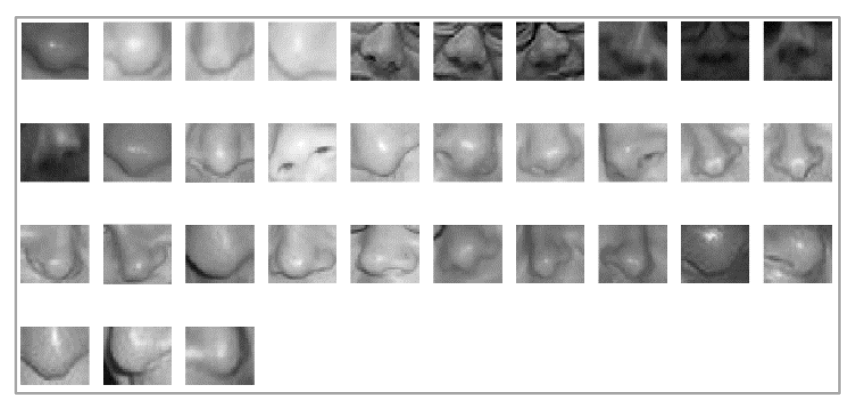

Fig:5) Nose Template

Retrieval Number: D6689049420/2020@BEIESP
The algorithm for the proposed method given below,

Step 1: Create a template for eyes, nose, mouth, and face

Step 2: Accept an input image as a target image.

Step 3: Calculate normalized cross-correlation (NCC) value of target and template image for eyes, nose, and mouth.

Step 4: If NCC value $\ngtr 0.99$ then rotate $(0-360 \circ)$ the template at a certain angle interval and check the NCC value.

Step 5: If NCC values $>0.99$ then draw a boundary box at the matched area.

Step 6: If eye, nose, and mouth any of the two found, means if the NCC value for eyes, nose, and mouth $>0.99$, then check the NCC value for the face. If NCC values for face $>0.99$ that means the face and the facial parts of that face found.

Step 7: If the eye, nose, and mouth any of the two found, means if the NCC value for eyes, nose, and mouth $<0.99$, then print no face found.

The diagram for the proposed algorithm is given below:

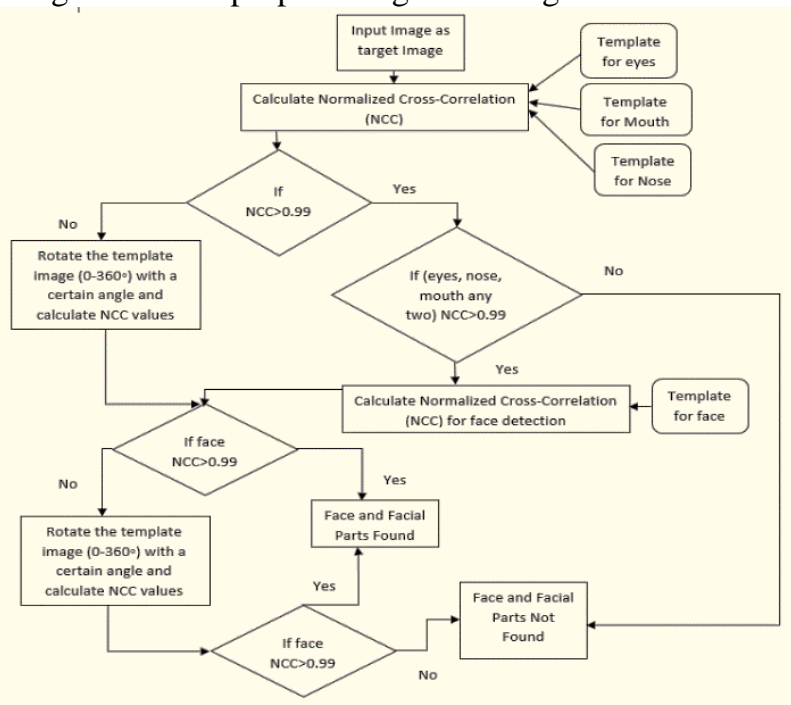

Flowchart of the Proposed Algorithm

II. EXPERIMENTAL RESULT:

The sample of the face template shown below:

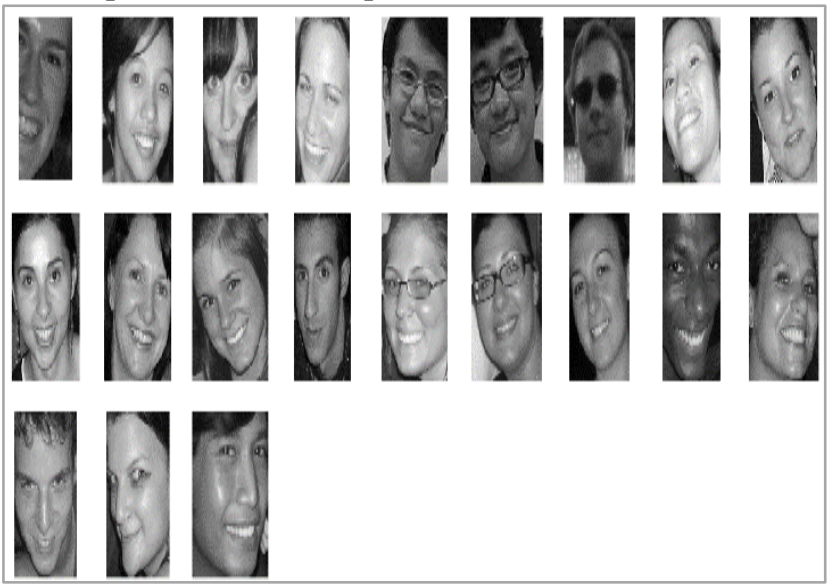

Fig: 6) Face Template

After detecting the face the result is given below,

Published By:

Blue Eyes Intelligence Engineering

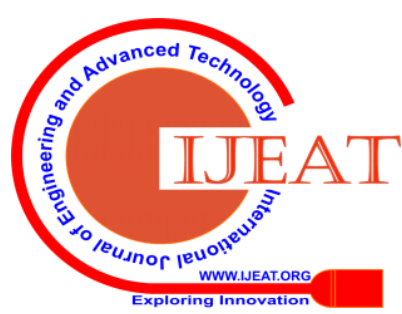



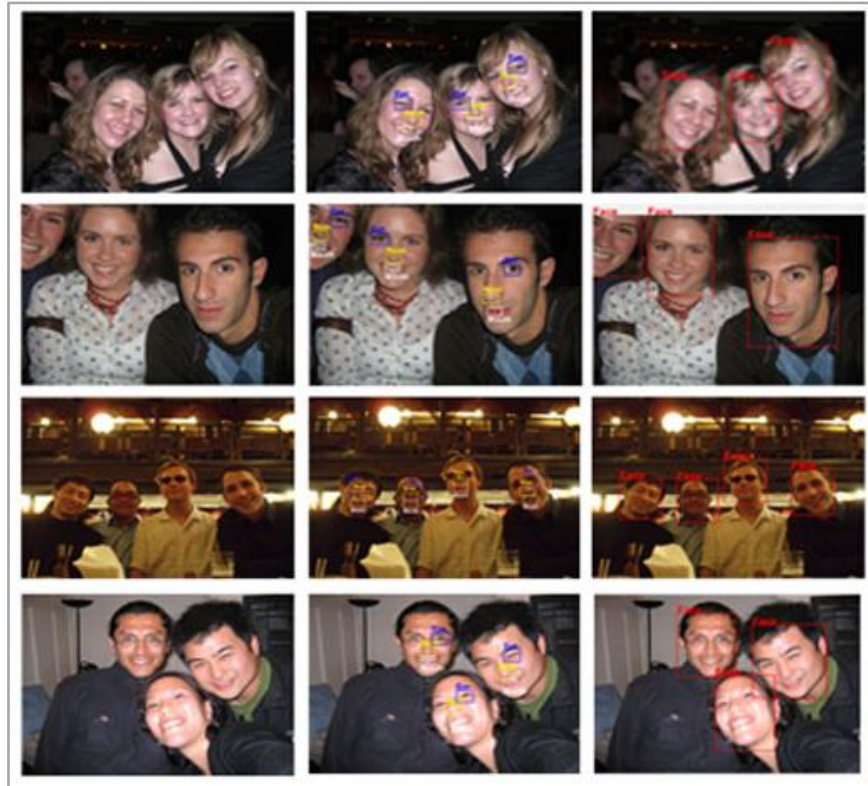

C

Fig: 7) a) uriginal Image, b) Atter detecting various facial parts [nose, eyes, mouth], c) After Detecting Face

Here more than 100 images are used to verify the algorithm. In this proposed method, it is needed to convert the target and template image into greyscale images and the template must the same or less size with respect to the target images. According to the proposed method, the limitations of this method are if eyes covered with color glasses cannot be identified and also the side faces where eyes nose and mouth not properly identified, no able identified the face. Generally, the template matching used to identify the image parts where it can work well with the images, regardless of the intensity of the image, the size of the image, etc. The main advantage of this method is that it can identify the proper and accurate region of matching.

\section{CONCLUSION}

In this, a pattern recognition technique used to identify the human faces along with the various facial parts. The Normalized Cross-Correlation technique used here to detect the faces and its facial parts. It is one of the efficient techniques to detect multiple faces in a group of people images.

Generally, the correlation template matching technique takes time to detect, but this algorithm is fast while maintaining high accuracy. Finally, a GUI was created to make this whole process easier for all users.

\section{REFERENCES}

1. Oza, Shrinath, et al. "Facial Recognition Using Deep Learning Neural Network." International Journal for Research in Applied Science \& Engineering Technology (IJRASET), Dec. 2017, www.ijraset.com. ISSN: 2321-9653; IC Value: 45.98; SJ Impact Factor:6.887; Volume 5, Issue XII

2. CHOI, SANG-IL, et al. "Face Recognition Using Composite Features Based on Discriminant Analysis." Digital Object Identifier 10.1109/ACCESS.2018.2812725, 6 Mar. 2018, ieexplore.ieee.org/iel7/6287639/6514899/08307164.pdf.

3. Wang, Ru, and Xinshi He. "Face Detection Based on Template Matching and Neural Network." 2019 International Conference on Communications, Information System and Computer Engineering (CISCE), 2019, doi:10.1109/cisce.2019.00127.

Published By:

Blue Eyes Intelligence Engineering

\& Sciences Publication 10.1109/ccdc.2018.8408105.
4. Singh, Namrata, et al. "Template Matching for Detection \& Recognition of Frontal View of Human Face through Matlab." 2017 International Conference on Information Communication and Embedded Systems (ICICES), 2017, doi:10.1109/icices.2017.8070792.

5. Yan, Chen, et al. "Multi-Angle Face Detection Based on Gaussian Mixture Model and Improved Template Matching." 2018 Chinese Control And Decision Conference (CCDC), 2018, doi:

6. Agung, Nur, et al. "Frontal View Face Detection on Color Image Processing Using Template Matching Method." International Journal of Computer Applications, vol. 180, no. 35, 2018, pp. 31-36., doi:10.5120/ijca2018916880.

7. Khalil, M, and Ahmed Ibrahim. "Quick Techniques for Template Matching by Normalized Cross-Correlation Method." British Journal of Mathematics \& Computer Science, vol. 11, no. 3, 2015, pp. 1-9., doi:10.9734/bjmcs/2015/16461.

8. Swaroop, Paridhi, and Neelam Sharma. "An Overview of Various Template Matching Methodologies in Image Processing." International Journal of Computer Applications, vol. 153, no. 10, 2016, pp. 8-14., doi:10.5120/ijca2016912165. 